\title{
BMJ Open A multicentre observational study on neonates exposed to SARS-CoV-2 in China: the Neo-SARS-CoV-2 Study protocol
}

Tiantian Xiao (1) ${ }^{1,2}$ Shiwen Xia, ${ }^{3}$ Linkong Zeng, ${ }^{4}$ Guang Lin, ${ }^{5}$ Qiufen Wei, ${ }^{6}$
Wei Zhou, ${ }^{7}$ Deyi Zhuang, ${ }^{8}$ Xiao Chen, ${ }^{9}$ Bin Yi, ${ }^{10}$ Long Li, ${ }^{11}$ Hongying Mi, ${ }^{12}$
Zhaoqing Yin, ${ }^{13}$ Xiuyong Cheng, ${ }^{14}$ Laishuan Wang, ${ }^{15}$ Xiaojing Hu, ${ }^{1}$ Wenhao Zhou ${ }^{1}$

To cite: Xiao T, Xia S, Zeng $\mathrm{L}$, et al. A multicentre observational study on neonates exposed to SARS-CoV- 2 in China: the Neo-SARS-CoV-2 Study protocol. BMJ Open 2020;10:e038004. doi:10.1136/ bmjopen-2020-038004

- Prepublication history and additional material for this paper are available online. To view these files, please visit the journal online (http://dx.doi. org/10.1136/bmjopen-2020038004).

Received 24 February 2020 Revised 25 June 2020 Accepted 03 July 2020

Check for updates

(c) Author(s) (or their employer(s)) 2020. Re-use permitted under CC BY-NC. No commercial re-use. See rights and permissions. Published by BMJ.

For numbered affiliations see end of article.

Correspondence to Professor Wenhao Zhou; zhouwenhao@fudan.edu.cn

\section{ABSTRACT}

Introduction An outbreak of severe acute respiratory syndrome coronavirus 2 (SARS-CoV-2) occurred in Wuhan, China starting in December 2019. Yet the clinical features and long-term outcomes of neonates with SARS-CoV-2 exposure are lacking. The purpose of this study is to describe the clinical course and prognosis of the neonates exposed to SARS-CoV-2.

Methods and analysis This is a multicentre observational study conducted at the designated children and maternal and child hospitals in the mainland of China. Neonates exposed to SARS-CoV-2 infection will be recruited. The data to be collected via case report forms include demographic details, clinical features, laboratory and imaging results, as well as outcomes. Primary outcomes are the mortality of neonates with COVID-19 and SARSCoV-2 infection of neonates born to mothers with COVID-19. Secondary outcomes are the birth weight, premature delivery and neurological development of neonates exposed to SARS-CoV-2. The neurological development is assessed by the Chinese standardised Denver Developmental Screening Test at the corrected age of 6 months.

Ethics and dissemination This study has been approved by the Children's Hospital of Fudan University ethics committee (No. (2020)31). The study findings will be disseminated in peer-reviewed journals and presented at national and international conferences in order to improve the understanding of the clinical course among neonates exposed to SARS-CoV-2 and to provide evidence-based treatment and prevention strategies for this group. Trial registration number NCT04279899.

\section{INTRODUCTION}

Since December 2019, there has been an outbreak of severe acute respiratory syndrome coronavirus 2 (SARS-CoV-2) infection in Wuhan, China. It is genetically different from severe acute respiratory syndrome coronavirus (SARS coronavirus, SARS-CoV) and Middle East respiratory distress syndrome coronavirus (MERS coronavirus, MERS-CoV). ${ }^{1}$ The WHO has officially named the disease
Strengths and limitations of this study

- This is the first study to describe the neurological development of neonates exposed to SARS-CoV-2.

- Data are collected prospectively and systematically using a predesigned standardised case report form in order to minimise the information bias.

- The majority of the data are from Hubei province, so the generalisability may be limited.

- The diagnosis of affected status of COVID-19 for the maternal and neonatal cases were based on reverse transcription PCR tests, which cannot avoid the chance of misclassification.

as COVID-19. As of 20 February 2020, SARS-CoV-2 has affected 74675 persons and has caused more than 2121 deaths in China, and it is rapidly spreading across the world. The recent research of Wang et al has indicated that the higher affinity of SARS-CoV-2 spike binding to ACE2 receptor compared with SARS-CoV leads to its rapid spread across the world. ${ }^{2}$ Studies identified that patients with COVID-19 were infected though personto-person transmission, and most patients with COVID-19 travelled to Wuhan within 14 days before the disease onset; also a familial aggregation was observed. ${ }^{3}$ The main transmission is respiratory droplets, but it can also be transmitted through close contact. Recent studies have suggested that SARS-CoV-2 may be transmitted through the fecal-oral route. ${ }^{4}$ However, the maternal-fetal vertical transmission remains controversial. Two recent studies showed that there were no clinical findings or investigations suggestive of COVID-19 among neonates born to infected pregnant women and all samples, including amniotic fluid, cord blood and breast milk were detected negative for SARS-CoV-2. ${ }^{5}{ }^{6}$ Moreover, none of the neonates born to pregnant women with 
SARS-CoV got infected through vertical transmission. ${ }^{7}$ Recent research showed that there was low expression of the receptor ACE2, which has been identified as the functional receptor for SARS-CoV-2-induced lung injury, in the different cell types of the maternal-fetal interface of placentas from 6 gestational weeks to 14 gestational weeks. ${ }^{8}$ The study suggested that the possibility of vertical transmission of infection via placenta was low in early pregnancy. ${ }^{8}$ However, one neonate with a positive SARS-CoV-2 test at age 36 hours, born to the mother with COVID-19, has been reported by the National Health Commission. Notably, the total sample sizes from the current observational studies on affected pregnant women and neonates are small. ${ }^{56}$

The clinical manifestations of COVID-19 are classified according to the presence or absence of apparent symptoms, ranging from asymptomatic infection to severe pneumonia with acute respiratory distress syndrome, septic shock, acute kidney injury, disseminated intravascular coagulation, rhabdomyolysis and multiorgan failure leading to death. ${ }^{9} 10$ The most common symptoms are fever $(87.9 \%)$ and cough $(67.7 \%) .{ }^{10}$ The clinical shortterm outcomes are associated with oxygen saturation, respiratory rate, blood leucocyte/lymphocyte count and chest X-ray/CT findings. ${ }^{9} 10$ Although the pregnant women and children are susceptible to SARS-CoV-2 in the early phase of the COVID-19 epidemic, ${ }^{11}$ the clinical course of children with COVID-19 was less aggressive compared with that of adults. ${ }^{9} 1012$ The symptoms of the confirmed children include fever, cough, tachypnoea, vomiting and diarrhoea, which are flu-like symptoms. ${ }^{12}$ However, thus far, few neonates infected with SARS-CoV-2 have been reported. ${ }^{13}$

In addition, the study by Zhu et al has shown that two newborns presented with elevated procalcitonin (PCT, reference value: $<0.05 \mathrm{ng} / \mathrm{mL}$ within 6 hours of life) and/or interleukin 6 (IL-6, reference range: $0-20.9 \mathrm{pg}$ / $\mathrm{mL}$ ) within 72 hours of life (PCT $9.2 \mathrm{ng} / \mathrm{mL}$, IL-6 $274.6 \mathrm{pg} / \mathrm{mL}$, in one; PCT $20.74 \mathrm{ng} / \mathrm{mL}$, in another), and one of these died of multiple organ failure. ${ }^{5}$ It is known that prenatal infection/inflammation predisposes to fetal inflammatory response syndrome (FIRS) ${ }^{14}$ Therefore, the elevated inflammatory markers of the two newborns after birth may be related to the maternal COVID-19. Francis et al reported that neonates with FIRS had lower mean motor development scores than those without FIRS. ${ }^{15}$ Moreover, animal and clinical studies have indicated that human coronaviruses have been related to central nervous system pathogenic processes, such as demyelinating disease in humans. ${ }^{16}$ Human coronavirus in cerebrospinal fluid has been identified as the pathogen in a child with acute disseminated encephalomyelitis. ${ }^{17}$ Currently, no studies have described the neurological outcomes of neonates with COVID-19. Additionally, SARS-CoV and MERS-CoV have caused perinatal adverse outcomes, including fetal distress, fetal growth restriction, preterm birth and fetal demise. ${ }^{18-20}$ Compared with the clinical features of SARS-CoV and MERS-CoV, the rate of adverse outcomes of SARS-CoV-2-infected mothers and fetal mortality seems lower. ${ }^{5620}$ For these reasons, we have undertaken this observational study focusing on the clinical course and long-term neurological outcomes of neonates with SARS-CoV-2 exposure.

\section{OBJECTIVES}

The aim of the study is to describe the clinical findings and long-term neurological outcomes of neonates exposed to SARS-CoV-2 and to evaluate the clinical characteristics and prognosis of neonates born to mothers with COVID-19.

\section{METHODS AND ANALYSIS}

Study design and setting

This observational study obtains clinical data from designated children and maternal and child hospitals in 31 provinces/municipalities of the mainland of China from 1 February 2020 to 31 November 2020. Given that SARS-CoV-2 spreads rapidly and is contagious to the general population, all suspected or confirmed patients are admitted to designated hospitals. Patients are enrolled in this study after receiving informed consent from the patient or their guardian. The medical information questionnaires are classified into maternal and neonatal versions, which include demographic details, epidemiological history, clinical manifestations, timing and results of laboratory tests and imaging, and therapeutic data. Two researchers independently check and record the data to ensure accuracy. Each maternal and neonatal case report form (CRF) is assigned a unique identifier number and the initials of the first name and family name of the patient to maintain confidentiality (see CRFs in online supplementary file).

Eligible infants will be followed up at five time points no matter whether the infants are in hospital or discharged at home, which are days 3, 7, 14, 28 after enrolment and the corrected age of 6 months. If the infants are discharged at home, they will be followed up at the clinic of the designated hospitals. If the infant is dead during initial hospitalisation, the next few follow-ups will not be conducted. The neonates born at more than 35 weeks +0 days are followed up at 6 months after birth and the infants born at less than 35 weeks +0 days are followed up at a corrected age of 6 months (see figure 1 ).

\section{Study population}

Study participants are being recruited from all the designated children and maternal and child hospitals in China during the study period. Considering the emerging disease, our study used a convenience sampling method, and all eligible participants from a large number of study centres were consecutively recruited, although not randomly, thus selection bias has been minimised.

\section{Criteria for patient inclusion}

1. Age from day 0 to 28 days. 


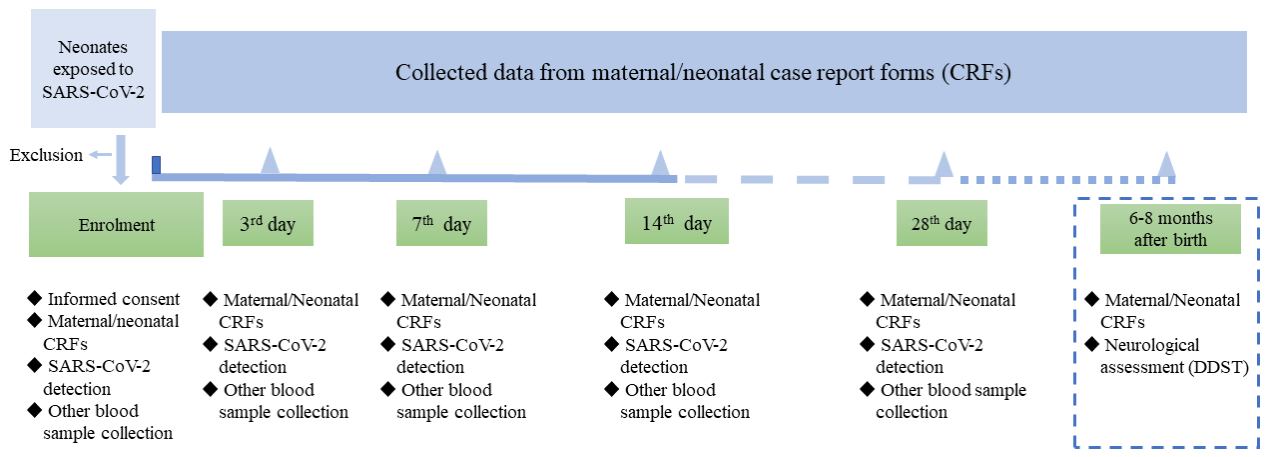

Figure 1 Protocol and follow-up of neonates exposed to severe acute respiratory syndrome coronavirus 2 (SARS-CoV-2). Regarding the enrolment time point, the neonates born to mothers with COVID-19 are enrolled on the day of birth and the neonates with COVID-19 are enrolled on the day of admission. DDST, Denver Developmental Screening Test.

2. Neonates with COVID-19, or neonates born to mothers with COVID-19.

\section{Criteria for patient exclusion}

Neonates with major anomalies. The major anomalies are defined according to the US Centers for Disease Control and Prevention (CDC) guidelines. ${ }^{21}$

\section{Sample size}

This study will comprise all the participants from all of the designated children and maternal and child hospitals in the mainland of China during the study period. A minimum of 100 individuals will be recruited.

\section{Study measures}

Primary outcomes are mortality of neonates with COVID-19 at the time of initial discharge and SARS-CoV-2 infection of neonates born to mothers with COVID-19 within 7 days of birth. The diagnosis of neonatal COVID-19 is based on the guidelines provided by the National Health Commission and the Chinese Perinatal-Neonatal SARS-CoV-2 Committee. $^{22} 23$

Secondary outcomes are listed below:

1. The birth weight of the neonates born to mothers with COVID-19 at birth.

2. Preterm delivery of neonates born to mothers with COVID-19 at delivery.

3. The disease severity of neonates with COVID-19 during initial hospitalisation. $^{22}$

4. The neurological development measured by the Chinese standardised Denver Developmental Screening Test (DDST) in neonates exposed to SARS-CoV-2. ${ }^{24}$

Neonates with SARS-CoV-2 infection are classified into asymptomatic, mild infection and severe infection, according to the expert consensus provided by the Chinese Perinatal-Neonatal SARS-CoV-2 Committee. ${ }^{22}$

The DDST is one of the most prevalent screening tools used for children aged 1 month to 6 years. ${ }^{24}{ }^{25}$ The standardised DDST consists of 104 items and covers four areas of development: (1) Personal/social. (2) Fine motor/adaptive. (3) Language. (4) Gross motor. In the study, three trained professionals examine the children.
The results of DDST could be normal (no delays), suspect (two or more caution items and/or one or more delays), abnormal (two or more delays) or untestable (refusal of one or more items completely to the left of the age line or more than one item intersected by the age line in the $75 \%-90 \%$ area). The children with abnormal results are retested in 2-3 weeks. The infants born at more than 35 weeks+0 days are evaluated at 6 months after birth and the infants born at less than 35 weeks+0 days are evaluated at a corrected age of 6 months.

The study explores other clinical variables, including age, gender, the epidemiological history, clinical manifestations (fever, lethargy, cough, vomiting, oxygen saturation, etc), collection time and results of laboratory testing, time and results of imaging, and therapeutic data (see CRFs in online supplementary file).

\section{Laboratory processing}

SARS-CoV-2 testing

In this study, SARS-CoV-2 will be tested in blood, cord blood, amniotic fluid, placenta, respiratory tract, stool, urine, breast milk from mothers or neonates as scheduled if the samples are available (see figure 1). SARS-CoV-2 will be tested by real-time reverse transcription PCR (RT-PCR) in the Chinese CDC. The collection, transfer, processing and testing of samples meet WHO requirements. According to WHO's RT-PCR guidelines, CDC uniformly uses the kit recommended by the Chinese CDC (BioGerm, Shanghai, China) to detect SARS-CoV-2 in the samples from mothers and newborns.

\section{Other examinations}

The completed blood count, $\mathrm{C}$ reactive protein, PCT, blood gas analysis, acid-base studies, serum electrolyte and creatinine measurements, blood urea nitrogen, liver function tests, cardiac biomarkers, coagulation function and chest imaging are performed when clinically indicated in the enrolled hospitals. Tests for other aetiology of infection, such as influenza viruses, respiratory syncytial virus, bacteria, and so on, are performed if clinically indicated (see CRFs in online supplementary file). 


\section{Data analysis plan}

Descriptive statistics will be used to define clinical parameters in neonates exposed to SARS-CoV-2. For continuous variables, values and SD will be reported; for categorical variables, percentages and $\mathrm{SD}$ will be reported. The Student's t-test for independent groups will be used for data with a normal distribution. The differences in categorical variables will be measured by the $\chi^{2}$ test or Fisher's exact test, and the differences in abnormally distributed quantitative variables will be analysed by the MannWhitney $\mathrm{U}$ test. The analyses will be primarily conducted using SPSS V.20.

\section{Ethics and dissemination}

Any protocol modifications will be communicated to the study team and ethics committees. This study has been approved by the Children's Hospital of Fudan University ethics committee. Written informed consent is obtained from all participants. For neonates, written informed consent will be obtained from their parents/ legal guardians. The research team will explain the study information to the neonate's mother/guardian orally or in writing, including the study objectives, potential risks and benefits, inconveniences and the participants' rights and responsibilities. The investigating team will write all articles, and submit them for peer-reviewed publication; authorship inclusion and order will be measured by levels of contribution.

\section{Study status}

The study began recruitment on 1 February 2020. Thus far, 43 maternal-neonatal pair have been recruited. Among these, three neonates born to mothers with COVID-19 have been tested positive for SARS-CoV-2 in nasopharyngeal samples within 72 hours after birth and no deaths have been reported. ${ }^{26}$

\section{Patient and public involvement}

There are no patients and public involved in the study.

\section{Author affiliations}

${ }^{1}$ Department of Neonatology, Children's Hospital of Fudan University, Shanghai, China

${ }^{2}$ Department of Neonatology, Chengdu Women's and Children's Central Hospital, School of Medicine, University of Electronic Science and Technology of China, Chengdu, China

${ }^{3}$ Department of Neonatology, Hubei Maternal and Child Health Hospital, Wuhan, China

${ }^{4}$ Department of Neonatology, Wuhan Children's Hospital, Wuhan, China

${ }^{5}$ Department of Pediatrics, Zhu Hai Maternal and Children's Hospital, Zhuhai, China

${ }^{6}$ Department of Neonatology, The Maternal and Child Health Hospital of Guangxi

Zhuang Autonomous Region, Guangxi Zhuang Autonomous Region, China

${ }^{7}$ Department of Neonatology, Guangzhou Women and Children's Medical Centre, Guangzhou Medical University, Guangzhou, China

${ }^{8}$ Department of Pediatrics, Xiamen Children's Hospital, Xiamen, China

${ }^{9}$ Department of Pediatrics, First Affiliated Hospital of Nanchang University,

Nanchang, Jiangxi, China

${ }^{10}$ Department of Neonatology, Gansu provincial maternity and child-care hospital, Lanzhou, China

${ }^{11}$ Department of Neonatology, People's Hospital of Xinjiang Your Autonomous Region, Xinjiang, China
${ }^{12}$ Department of Neonatology, The First People's Hospital Of Yunnan Province, Kunming, China

${ }^{13}$ Department of Neonatology, Dehong People Hospital, Dehong, China

${ }^{14}$ Department of Neonatology, The First Affiliated Hospital of Zhengzhou University, Zhengzhou, China

${ }^{15}$ Department of Pediatrics, Children's Hospital of Fudan University, Shanghai, China

Acknowledgements The authors thank Prof Weili Yan, Yin Wang (PhD) and YI Zhang (MPh), from the Department of Clinical Epidemiology, Children's Hospital of Fudan University, Shanghai, China for designing the maternal and neonatal case report forms. The authors also thank all the physicians and nurses, parents and their infants, for their support in developing this research project.

Contributors WZH conceived this project. WZH, TX planned the statistical analyses. The first draft of the manuscript was written by TX and revised by WZH; WZH, TX, SX, LZ, GL, QW, WZ, DZ, XC, BY, LL, HM, ZY, XYC, LW, XH edited and reviewed the manuscript. It was approved by WZH, TX, SX, LZ, GL, QW, WZ, DZ, XC, BY, LL, HM, ZY, XYC, LW, XH.

Funding The authors have not declared a specific grant for this research from any funding agency in the public, commercial or not-for-profit sectors.

Competing interests None declared.

Patient and public involvement Patients and/or the public were not involved in the design, or conduct, or reporting, or dissemination plans of this research.

Patient consent for publication Obtained.

Provenance and peer review Not commissioned; externally peer reviewed.

Open access This is an open access article distributed in accordance with the Creative Commons Attribution Non Commercial (CC BY-NC 4.0) license, which permits others to distribute, remix, adapt, build upon this work non-commercially, and license their derivative works on different terms, provided the original work is properly cited, appropriate credit is given, any changes made indicated, and the use is non-commercial. See: http://creativecommons.org/licenses/by-nc/4.0/.

ORCID iD

Tiantian Xiao http://orcid.org/0000-0002-8045-9190

\section{REFERENCES}

1 Chen Y, Liu Q, Guo D. Emerging coronaviruses: genome structure, replication, and pathogenesis. J Med Virol 2020;92:418-23.

2 Wrapp D, Wang N, Corbett KS, et al. Cryo-EM structure of the 2019nCoV spike in the prefusion conformation. Science 2020;367:1260-3.

3 Chan JF-W, Yuan S, Kok K-H, et al. A familial cluster of pneumonia associated with the 2019 novel coronavirus indicating personto-person transmission: a study of a family cluster. Lancet 2020;395:514-23.

$4 \mathrm{Xu} \mathrm{Y,} \mathrm{Li} \mathrm{X,} \mathrm{Zhu} \mathrm{B,} \mathrm{et} \mathrm{al.} \mathrm{Characteristics} \mathrm{of} \mathrm{pediatric} \mathrm{SARS-CoV-2}$ infection and potential evidence for persistent fecal viral shedding. Nat Med 2020;26:502-5.

5 Zhu H, Wang L, Fang C, et al. Clinical analysis of 10 neonates born to mothers with 2019-nCoV pneumonia. Trans/ Pediatr 2020;9:51-60.

6 Chen H, Guo J, Wang C, et al. Clinical characteristics and intrauterine vertical transmission potential of COVID-19 infection in nine pregnant women: a retrospective review of medical records. Lancet 2020;395:809-15.

7 Li AM, Ng PC. Severe acute respiratory syndrome (SARS) in neonates and children. Arch Dis Child Fetal Neonatal Ed 2005;90:F461-5.

8 Zheng QL, Duan T, Jin LP. Single-cell RNA expression profiling of ACE2 and Axl in the human maternal-Fetal interface. Available: http://www.repdevmed.org/preprintarticle.asp?id=278679.

9 Huang C, Wang Y, Li X, et al. Clinical features of patients infected with 2019 novel coronavirus in Wuhan, China. Lancet 2020;395:497-506.

10 Guan W-J, Ni Z-Y, Hu Y, et al. Clinical characteristics of coronavirus disease 2019 in China. N Engl J Med 2020;382:1708-20.

11 Liu W, Zhang Q, Chen J, et al. Detection of Covid-19 in children in early January 2020 in Wuhan, China. N Engl J Med 2020;382:1370-1.

12 Wei M, Yuan J, Liu Y, et al. Novel coronavirus infection in hospitalized infants under 1 year of age in China. JAMA 2020. doi:10.1001/ jama.2020.2131. [Epub ahead of print: 14 Feb 2020].

13 Zeng LK, Tao XW, Yuan WH, et al. [First case of neonate infected with novel coronavirus pneumonia in China]. Zhonghua Er Ke Za Zhi 2020;58:E009. 
14 Musilova I, Andrys C, Drahosova M, et al. Late preterm prelabor rupture of fetal membranes: fetal inflammatory response and neonatal outcome. Pediatr Res 2018;83:630-7.

15 Francis $\mathrm{F}$, Bhat $\mathrm{V}$, Mondal $\mathrm{N}$, et al. Fetal inflammatory response syndrome (FIRS) and outcome of preterm neonates - a prospective analytical study. J Matern Fetal Neonatal Med 2019;32:488-92.

16 Desforges M, Le Coupanec A, Dubeau P, et al. Human coronaviruses and other respiratory viruses: underestimated opportunistic pathogens of the central nervous system? Viruses 2019;12:14.

17 Yeh EA, Collins A, Cohen ME, et al. Detection of coronavirus in the central nervous system of a child with acute disseminated encephalomyelitis. Pediatrics 2004;113:e73-6.

18 Wong SF, Chow KM, Leung TN, et al. Pregnancy and perinatal outcomes of women with severe acute respiratory syndrome. Am J Obstet Gynecol 2004;191:292-7.

19 Alfaraj SH, Al-Tawfiq JA, Memish ZA. Middle East Respiratory Syndrome Coronavirus (MERS-CoV) infection during pregnancy: Report of two cases \& review of the literature. J Microbiol Immunol Infect 2019;52:501-3.

20 Shek CC, Ng PC, Fung GPG, et al. Infants born to mothers with severe acute respiratory syndrome. Pediatrics 2003;112:e254.
21 Rasmussen SA, Olney RS, Holmes LB, et al. Guidelines for case classification for the National birth defects prevention study. Birth Defects Res A Clin Mol Teratol 2003;67:193-201.

22 Wang $\mathrm{L}$, Shi $\mathrm{Y}, \mathrm{Xiao} \mathrm{T}$, et al. Chinese expert consensus on the perinatal and neonatal management for the prevention and control of the 2019 novel coronavirus infection (first edition). Ann Transl Med 2020;8:47.

23 China NHC. New coronavirus pneumonia prevention and control protocol. 5 edn. National Health Commission of the People's Republic of China, 2020. http://www.nhc.gov.cn/xcs/fkdt/202002/ e84bd30142ab4d8982326326e4db22ea.shtml

24 Xie Z-H, Bo S-Y, Zhang X-T, et al. Sampling survey on intellectual disability in 0 approximately 6 -year-old children in China. J Intellect Disabil Res 2008;52:1029-38.

25 Committee on Children With Disabilities. Developmental surveillance and screening of infants and young children. Pediatrics 2001;108:192-5.

26 Zeng L, Xia S, Yuan W, et al. Neonatal early-onset infection with SARS-CoV-2 in 33 neonates born to mothers with COVID-19 in Wuhan, China. JAMA Pediatr 2020;174:722. 